\title{
A Note on the Disturbed Equations of Motion in the Non-Holonomic Coordinates.
}

\author{
DJoRdJe S. DJukró (Novi Sad, Jugoslavia) (*)
}

Summary. - The aim of this artiole is to establish the disturbed equations of motion in the nonholonomic coordinates. The governing differential equations of non disturbed motion are the Hamel-Boltzman equations. The disturbed equations obtained in this article can be used for consideration in holonomie and non-holonomic dynamics systems.

\section{1. - Introduction.}

In many physical cases it is more convenient to study the problem under consideration not by original coordinate but by non-holonomic coordinates (see for example [7], [8], [9], [10]). In the present literature there are not general approach about stability of motion in the non-holonomic coordinates (see for example [1], [2], [3], exspecially, for exaustive review, paper [1]).

In the paper the following conventions will be observed:

1) Greek indices $\alpha, \beta$ and $\gamma$ unrepeated imply a range of values from $m+1$ to $n$, and, when repeated in a single term, summation over that range.

2) Small italic indices $a, b$ and $c$ unrepeated imply a range of values from 1 to $m$, and, when repeated in a single term, summation over that range.

3) All another indices, greek or italie, takes values from 1 to $n$. The summation convention is again employed throughout.

Let us consider a holonomic mechanical system of $n$ degrees of freedom. If the $q^{i}$ are regarded as the generalized coordinates, and if $t$ is the time, for a large class of dynamical systems, the kinetic energy is given by a quadratic form of the generalized velocity $\dot{q}^{i}$

$$
T=\frac{1}{2} \boldsymbol{a}_{r s} \dot{q}^{r} \dot{q}^{s}
$$

where $a_{r s}$ is the fundamental metric tensor of Riemannian configuration space $V_{n}$.

Let us study the problem not by original velocities $\dot{q}^{i}$, but by their non-integrable linear combinations

$$
(1)^{\sigma}=\varphi_{i}^{\sigma} \dot{q}^{i}
$$

(*) Entrata in Redazione l'8 maggio 1972. 
where $\varphi_{i}^{\alpha}$ are given functions of coordinates $q^{i}$. The inverse expression of $(2)$ is

$$
\dot{q}^{i}=\varphi_{\sigma}^{i} \omega^{\sigma},
$$

where $\varphi_{\sigma}^{i} \psi_{j}^{\sigma}=\delta_{j}^{i}$ and $\varphi_{0}^{i} \varphi_{i}^{\sigma}=\delta_{0}^{\sigma}$. Putting (3) into the expression of kinetic energy we have

$$
\widetilde{T}=\frac{1}{2} g_{\sigma \mu} \omega^{\sigma} \omega^{\mu}
$$

where

$$
a_{\sigma \mu}=a_{r_{s}} \varphi_{\sigma}^{r} \varphi_{\mu}^{s}
$$

is the metric tensor with respect to the non-holonomic coordinates. With (2) can be connected the $n$ linear differential forms

$$
d \pi^{\sigma}=\varphi_{i}^{\sigma} d q^{i}
$$

where $d \pi^{\sigma}$ are differentials of non-holonomic coordinates.

Explicit form of the Hamel-Boltzman's equations of motion, for the dynamical systems with the kinetic energy (4), are (see [7], p. 176)

$$
\dot{\omega}^{*}+A_{Q \sigma}^{\kappa} \omega^{\varrho}()^{\sigma}=\Pi^{x}
$$

Here,

$$
A_{e \sigma}^{\mu}=I_{e \sigma}^{x}+g^{\mu v} g_{q \varrho} \gamma_{\sigma \nu}^{\tau},
$$

are coefficients of connection in non-holonomic coordinates,

$$
\gamma_{\sigma p}^{\tau}=\varphi_{\sigma}^{p} \varphi_{v}^{r}\left(\frac{\partial \varphi_{p}^{\tau}}{\partial q^{r}}-\frac{\partial \varphi_{r}^{\tau}}{\partial q^{p}}\right)
$$

are so called objects of anholonomity,

$$
\Gamma_{v \varrho}^{l}=g^{l \sigma} \Gamma_{\sigma .2 \varrho}
$$

and

$$
\Gamma_{\sigma, \nu \varrho}=\frac{1}{2}\left(\frac{\partial g_{\sigma v}}{\partial \pi^{Q}}+\frac{\partial g_{g \sigma}}{\partial \pi^{v}}-\frac{\partial g_{v o}}{\partial \pi^{\sigma}}\right)
$$

are Christoffel's symbols of the second and first order with respect to the nonholonomic coordinates. If the generalized external forces, acting on the system, are $Q_{i}(t, q, \dot{q})$, than generalized forces with respect to the non-holonomic coordinates are

$$
\Pi_{v}=q_{v}^{i} Q_{i} .
$$


Putting (3) into (12) we have that $\Pi_{v}$ is the functions of $t, q^{i}$ and $\omega^{\sigma}$, i.e.

$$
\Pi_{p}=\Pi_{p}(t, q, \omega)
$$

It is known (see for example [7]), when the integrability conditions for system (6) are not filled, than it is impossible to found explicit functional dependence $\pi^{i}=$ $=\pi^{i}\left(q^{j}\right)$, or inverse $q^{i}=q^{i}\left(\pi^{j}\right)$. In such cases coefficients of the connections $A_{\ell \sigma}^{*}$ are the functions of non-holonomic coordinates $\pi^{i}$ only formaly. So, in this paper, will be assumed that coefficients $\Lambda_{\delta \sigma}^{\kappa}$ are the functions of generalized coordinates $q^{i}$.

\section{2. - The disturbed equations of motion in the non-holonomic coordinates.}

The equations of an undisturbed path $O$ of the representative single particle in the configuration space, and with respect to non-holonomic coordinates are (7). Let $q^{\sigma}$ be the coordinates of a point $P$ of $C$ and ${ }^{*} q^{\alpha}=q^{\alpha}+x^{\sigma}$ the coordinates of the corresponding point $* P$ of the disturbed path $* C, x^{\sigma}$ being infinitesimal. The vector $x^{\sigma}$ is the disturbance vector (see [2], p. 78). In a point $P$ of $C$ the representative particle have quasi-velocity $\omega^{*}$. In the corresponding point $* P$ of the disturbed path ${ }^{*} C$, the quasi-velocity vector have components ${ }^{*} \omega^{*}=\omega^{*}+\Omega^{*}$. We shall call vector $\Omega^{*}$ the disturbance vector of the quasi-velocity vector. The condition for stability of motion is that $x$ (the magnitude of the vector $x^{\alpha}$ ) and $\Omega$ (the magnitude of the vector $\Omega^{*}$ ) should remain permanently small.

Let us substitute ${ }^{*} \omega^{*}=\omega^{*}+\Omega^{*}$ in (7), since ${ }^{*} C$ is also the path of the representative particle, and obtain

$$
\dot{\omega}^{x}+\dot{\Omega}^{x}+* \Lambda_{\varrho \sigma}^{\varkappa}\left(\omega^{\theta}+\Omega^{o}\right)\left(\omega^{\sigma}+\Omega^{\sigma}\right)=* \Pi^{x}+\varepsilon \Phi^{*},
$$

where the asterisk indicates quantities to be calculated at $* P$. Here $\varepsilon$ is a small parameter and $\Phi^{x}(t, q, \omega)$ contravariant components of the external forces which are acting on the system only during the disturbed motion. Expanding quantities $* \Lambda_{e \sigma}^{\kappa}$ and $* I^{*}$, and retaining only first powers of small quantities we have

$$
\left\{\begin{array}{l}
* \Lambda_{o \sigma}^{*}=\Lambda_{o \sigma}^{*}+\frac{\partial \Lambda_{0 \sigma}^{\varkappa}}{\partial q^{\delta}} x^{\delta}, \\
* \Pi^{\varkappa}=\Pi^{\varkappa}+\frac{\partial \Pi^{\varkappa}}{\partial q^{\delta}} x^{\delta}+\frac{\partial \Pi^{\varkappa}}{\partial \omega^{\delta}} \Omega^{\delta} .
\end{array}\right.
$$

Combining (15), (14) and (7) we have

$$
\dot{Q}^{\varkappa}+x^{\delta} \omega^{e} \omega^{\sigma} \frac{\partial \Lambda_{\rho^{\sigma}}^{\varkappa}}{\partial q^{\delta}}+\Lambda_{q_{\sigma}}^{\varkappa}\left(\Omega^{\varrho} \omega^{\sigma}+\omega^{\varrho} \Omega^{\sigma}\right)=x^{\delta} \frac{\partial \Pi^{\varkappa}}{\partial q^{\delta}}+\Omega^{\delta} \frac{\partial \Pi^{\varkappa}}{\partial \omega^{\delta}}+\varepsilon \Phi^{\varkappa} .
$$


The disturbed quasi-velocities must also be in the consistent with the relations (2)

$$
{ }^{*} \omega^{\sigma}={ }^{*} \varphi_{i}^{\sigma *} \dot{q}^{i}
$$

or after expanding

$$
\omega^{\sigma}+\Omega^{\sigma}=\left(\varphi_{i}^{\sigma}+\frac{\partial \varphi_{i}^{\sigma}}{\partial q^{\delta}} x^{\delta}\right)\left(\dot{q}^{i}+\dot{x}^{i}\right)
$$

Taking in account only small quantities of the first order and using (2) we have

$$
\Omega^{\sigma}=\psi_{i}^{\sigma} \dot{x}^{i}+x^{\delta} \varphi_{Q}^{i} \omega^{Q} \frac{\partial \varphi_{i}^{\sigma}}{\partial q^{\delta}}
$$

This is the connections between the disturbances of the generalized coordinates $x^{i}$ and the disturbances of the quassi-velocity's components $\Omega^{\sigma}$. It is easy to get inverse relations of (19) (using $\varphi_{s}^{i} \varphi_{i}^{\sigma}=\delta_{j}^{i}$ )

$$
\dot{x}^{i}=\varphi_{\sigma}^{i} \Omega^{\sigma}+x^{\delta} \omega^{\sigma} \frac{\partial \varphi_{\sigma}^{i}}{\partial q^{\delta}} .
$$

The stability of motion can be estimated using $n$ differential equations (16) together with $n$ relations (19) or (20). As the solution of this systems we have $2 n$ quantities $\omega^{*}$ and $x^{i}$, i.e. the disturbances of the quassi-velocity vector and the disturbances of the generalized coordinates, as a functions of the time.

Let us consider a special case of the relations (2) defined with

$$
\psi_{i}^{\sigma}=\delta_{i}^{\sigma} ; \quad \varphi_{\sigma}^{i}=\delta_{\sigma}^{i}
$$

Than, using (2), (9), (8), (19), (12), (5), (10) and (11) we have

$$
\left\{\begin{array}{lll}
\omega^{\sigma}=\dot{q}^{\alpha} ; & \Pi_{\nu}=Q_{v} ; & \Omega^{\sigma}=\dot{x}^{\sigma} \\
\gamma_{\sigma \nu}^{\tau}=0 ; & g_{\sigma \mu}=a_{\sigma k} ; & A_{g \sigma}^{*}=\left\{\begin{array}{c}
\varkappa \\
\varrho \sigma
\end{array}\right\}
\end{array}\right.
$$

where $\left\{\begin{array}{c}x \\ \varrho\end{array}\right\}$ are Christoffel's symbols of the second order formed with respect to the fundamental metric tensor $a_{\sigma_{\mu}}$.

Combining (21), (22) and (16) we have

$$
\ddot{x}^{*}+2\left\{\begin{array}{c}
x \\
\varrho \sigma
\end{array}\right\} \dot{x}^{\rho} \dot{q}^{\alpha}+\dot{q}^{\alpha} \dot{q}^{\alpha} x^{\delta} \cdot \frac{\partial}{\partial q^{\delta}}\left\{\begin{array}{c}
x \\
\varrho \sigma
\end{array}\right\}-x^{\delta} \frac{\partial Q^{*}}{\partial q^{\delta}}=\dot{x}^{\delta} \frac{\partial Q^{*}}{\partial \dot{q}^{\delta}}+\varepsilon \Phi^{*}
$$

This differential equations are the original Synge's equations of the disturbances (compaire (23) with [2], p. 78 and [8], p. 623). 


\section{3. - The non-holonomic dynamical systems.}

Derived equations (16) and (19) can be used for the non-holonomic dynamical systems.

Let us suppose that a holonomic dynamical system is subjected to a $n-m$ non-holonomic constraints of the type

$$
\varphi_{i}^{\alpha} \dot{q}^{i}=0,
$$

where $\varphi_{i}^{\alpha}$ are a known functions of the generalized coordinates $q^{i}$.

The non-holonomic coordinates we will chose in such a way that $d \pi^{1}, \ldots, d \pi^{m}$ are independent, and another $n-m$ identicaly equal to zero (from the equations of the non-holonomic constraints)

$$
\dot{\tau}^{\alpha} \equiv \omega^{\alpha}=\varphi_{i}^{\alpha} \dot{q}^{i}=0 .
$$

For the disturbed motion we have that the velocities must satisfy the disturbed non-holonomic constraints, i.e.

$$
* \omega^{\alpha}=* \varphi_{i}^{\alpha *} \dot{q}^{i}=0
$$

Using (25), and remembering that $\omega^{\alpha}=\omega^{\alpha}+\Omega^{\alpha}$, we have that the disturbances of $n-m$ quassi-velocities are also equal to zero

$$
\Omega^{\alpha}=0
$$

Let be $R_{v}$ the generalized forces of constraints with respect to the non-holonomic coordinates. If this forces are ideal than we have that

$$
R_{a}=0 \quad \text { and } \quad R_{\alpha} \neq 0 .
$$

In this case the explicit form of the first $m$ equations of motion (7) is (see [7], p. 177)

$$
\dot{\omega}^{a}+\Lambda_{b c}^{a} \omega^{b} \omega^{c}=\Pi^{a} .
$$

From the last $n-m$ equations of the system (7) we can found the forces of the nonholonomic constraints

$$
R^{\alpha}=\Lambda_{c b}^{\alpha} \omega^{c} \omega^{b}-\Pi^{\alpha}
$$

Taking in account (27) and (25), we have, from (16) $m$ equations for the disturbances of the quassi-velocity's vector

$$
\dot{\Omega}^{a}+x^{\delta} \omega^{b} \omega^{c} \frac{\partial \Lambda_{b c}^{a}}{\partial q^{\delta}}+\Lambda_{b c}^{a}\left(\Omega^{b} \omega^{e}+\omega^{b} \Omega^{c}\right)=x^{\delta} \frac{\partial \Pi^{a}}{\partial q^{\delta}}+\Omega^{b} \frac{\partial \Pi^{a}}{\partial \omega^{b}}+\varepsilon \Phi^{a} .
$$

10 - Annali di Matematica 
This $m$ differential equations must be solved together with the non-holonomic constraints for the disturbances, which we get combining (19), (20), (25) and (27)

$$
\Omega^{a}=\varphi_{i}^{a} \dot{x}^{i}+x^{\delta} \varphi_{b}^{i} \omega^{b} \frac{\partial \varphi_{i}^{a}}{\partial q^{\delta}},
$$

or in the inverse form

$$
\dot{x}^{i}=\varphi_{a}^{i} \Omega^{a}+x^{\delta} \omega^{a} \frac{\partial \varphi_{a}^{i}}{\partial q^{\delta}}
$$

The systems (31) and (33) gives $m+n$ differential equations of the first order, from which, as solution, we have $m$ quantities $\Omega^{a}$ and $n$ quantities $x^{i}$ in the functional dependence of the time.

Combining last $n-m$ equations (16) with (27), (25), (28) and (30) we have the disturbances of the forces of the non-holonomic constraints in the form

$$
\delta R^{\alpha}=x^{\delta} \omega^{b} \omega^{c} \frac{\partial \Lambda_{b c}^{x}}{\partial q^{\delta}}+\Lambda_{b c}^{\alpha}\left(\Omega^{b} \omega^{c}+\omega^{b} \Omega^{e}\right)-x^{\delta} \frac{\partial \Pi^{x}}{\partial q^{\delta}}-\Omega^{a} \frac{\partial \Pi^{\alpha}}{\partial \omega^{\alpha}}-\varepsilon \Phi^{x}
$$

\section{4. - Equations for the magnitudes of the disturbance vector and the disturbance vector of the quasi-velocities.}

Let us introduce the unit disturbance vector $\mu^{r}$, with respect to the metric tensor $a_{r s}$ of the configuration Riemannian space $V_{n}$, and co-directional with $x^{r}$

$$
x^{r}=x \mu^{r}
$$

where

$$
a_{r s} \mu^{r} \mu^{s}=1 ; \quad x=\sqrt{a_{r s} x^{r} x^{s}}
$$

and the unit disturbance vector of the quasi-velocities $x^{\sigma}$, with respect to the metric tensor $g_{\sigma / k}$ of the non-holonomic space, and co-directional with $\Omega^{\sigma}$

$$
\Omega^{\alpha}=\Omega x^{\sigma}
$$

where

$$
g_{\sigma \mu} x^{\sigma} \chi^{\mu}=1, \quad \Omega=\sqrt{g_{\sigma \mu} \Omega^{\sigma} \Omega^{\mu}}
$$

Here $x$ is the magnitude of the disturbance vector and $\Omega$ is the magnitude of the disturbance vector of the quasi-velocities. Using (35), (36), (37), (38) and (3) 
DJORDJE S. DJUKIĆ: A note on the disturbed equations of motion, etc. 147

it is easy to obtain next relations

$$
\begin{aligned}
& \mu_{r} \dot{x}^{r}=\dot{x}-\frac{x}{2} \frac{\partial a_{r s}}{\partial q^{k}} \phi_{\sigma}^{k} \omega^{\sigma} \mu^{r} \mu^{s} \\
& \varkappa_{\sigma} \dot{\Omega}^{\sigma}=\dot{\Omega}-\frac{\Omega}{2} \frac{\partial g_{\sigma \mu}}{\partial q^{k}} \varphi_{y}^{k} \omega^{v} \varkappa^{\sigma} \varkappa^{\mu}
\end{aligned}
$$

where $\mu_{r}$ and $\varkappa_{\sigma}$ are covariant components of the vectors $\mu^{r}$ and $\varkappa^{\sigma}$.

Combining (39), (40), (16) and (20) we have

$$
\begin{gathered}
\varrho+I_{1} \Omega+I_{2} x=I_{3}, \\
\dot{x}+I_{4} x=I_{5} \Omega,
\end{gathered}
$$

where invariants have the forms

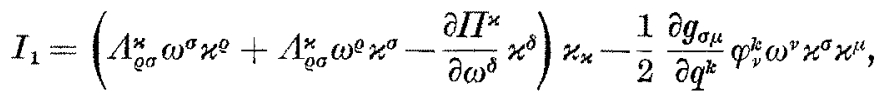

$$
\begin{aligned}
& I_{2}=\left(\frac{\partial \Lambda_{\rho_{\sigma}}^{x}}{\partial q^{\delta}} \omega^{\sigma} \omega^{\sigma}-\frac{\partial \Pi^{\star}}{\partial q^{\delta}}\right) \varkappa_{\kappa} \mu^{\delta}, \\
& I_{3}=\varepsilon \varkappa_{x} \Phi^{\varkappa}, \\
& I_{4}=-\frac{1}{2} \frac{\partial a_{r s}}{\partial q^{k}} \varphi_{a}^{k} \omega^{\alpha} \mu^{r} \mu^{s}-\omega^{\sigma} \mu_{i} \mu^{\delta} \frac{\partial \varphi_{Q}^{i}}{\partial q^{\delta}} \\
& I_{5}=\varphi_{\sigma}^{\zeta} \mu_{j} \varkappa^{\sigma} \text {. }
\end{aligned}
$$

Equations (41) and (42) may be called the invariant equations for the magnitudes of the disturbance vector and the disturbance of the quasi-velocity vector.

For the non-holonomic dynamical systems, in the presence of $n-m$ nonholonomic constraints (24), from (27) and (37), we have that

$$
x^{\alpha}=0 ; \quad x^{a} \neq 0
$$

Using (25), (27), (31), (33), (39), (40) and (48) we will get the invariant equations for non-holonomic dynamical system. These equations are of the same type as (41) and (42). The difference is only in the values of the invariants $(43) \div(47)$. The invariants for non-holonomic dynamical system we can get from $(43) \div(47)$ if we take in account $(48),(25),(27)$ and if we instead of the index $\psi$ write index $a$.

Acknowledgements. - The author thank Prof. B. VuJanovió for many useful discussions about this paper, and his useful comments on the manuscript. 


\section{REFERENCES}

[1] J. L. SYNGE, Geodesics in non-holonomic geometry, Math. Ann., 99 (1928), pp. 738-751.

[2] J. L. Sxnge, On the geometry of dynamies, Phil. Trans. Roy. Soc., A 226 (1926), pp. $31-106$.

[3] G. VRanchano, Sopra la stabilità geodetica, Rend. Acc. Lincei, 5 (1927), pp. 107-110.

[4] J. A. SchovтEN, Über nieht-holonome Übertragungen in einer $L_{32}$, Math. Zeitschr., 30 (1929), pp. 149-172.

[5] G. VRanceanu, Les espaces non-holonomes, Mém. des Sciences Mathém., Fase. LXXVI (1936).

[6] B. Voraxovic, The Hamiltonian Canonical Equations as a Problem in Non-Holonomic Coordinates, Proe. of the West Virginia Acad. of Scie., 41 (1969), pp. 324-328.

[7] V. V. DoBronravov, Osnovi mehaniki negolonomnih sistem, Izd., Visa skola, Moskva 1970 (in russian).

[8] A. I. LURJE, Analiticeshaja mehanika, Gosu. Iz. F.M. lit., Moskva, 1961 (in russian).

[9] J. I. Neimark - N. A. FufaEv, Dinamika negolonomnih sistem, Izd. Nauka, F.-M. Lit., Moskva, 1967 (in russian).

[10] E. T. WhITTAKER, A treatise on the analytical dynamics of particles and rigid bodies, University Press, Cambridge, 1965. 